\title{
D-Zero Cryobridge Structural Analysis
}

\author{
Engineering Note \#3740.510-EN-240
}

Issued: February 2, 1990

Appended: April 20, 1990

Originator: Jeff Western

Checked: Al Jaques

Appended: Al Jaques 


\section{D-Zero Cryobridge Structural Analysis * CG calculations and Lifting stresses apperded to Report on 4/6/9\%}

Engineering Note \#3740.510-EN-240

REF: DWG. No. $9220.740-M E-284211$ REN. C 2 SHTS (researen Div. Meen. Supp.)

Issued: April 6

Originator: Jeff Western

Checked: Al Jaques 


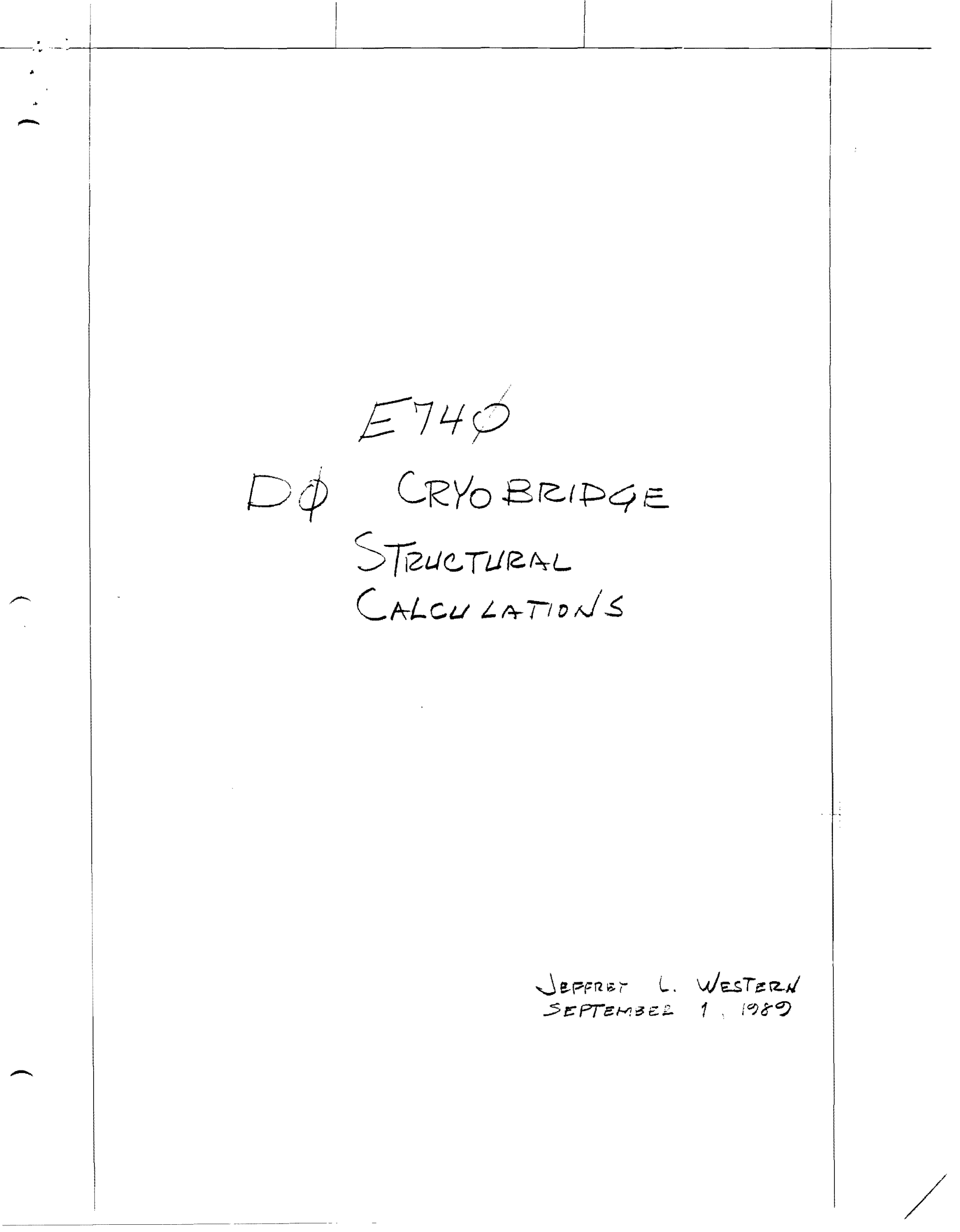




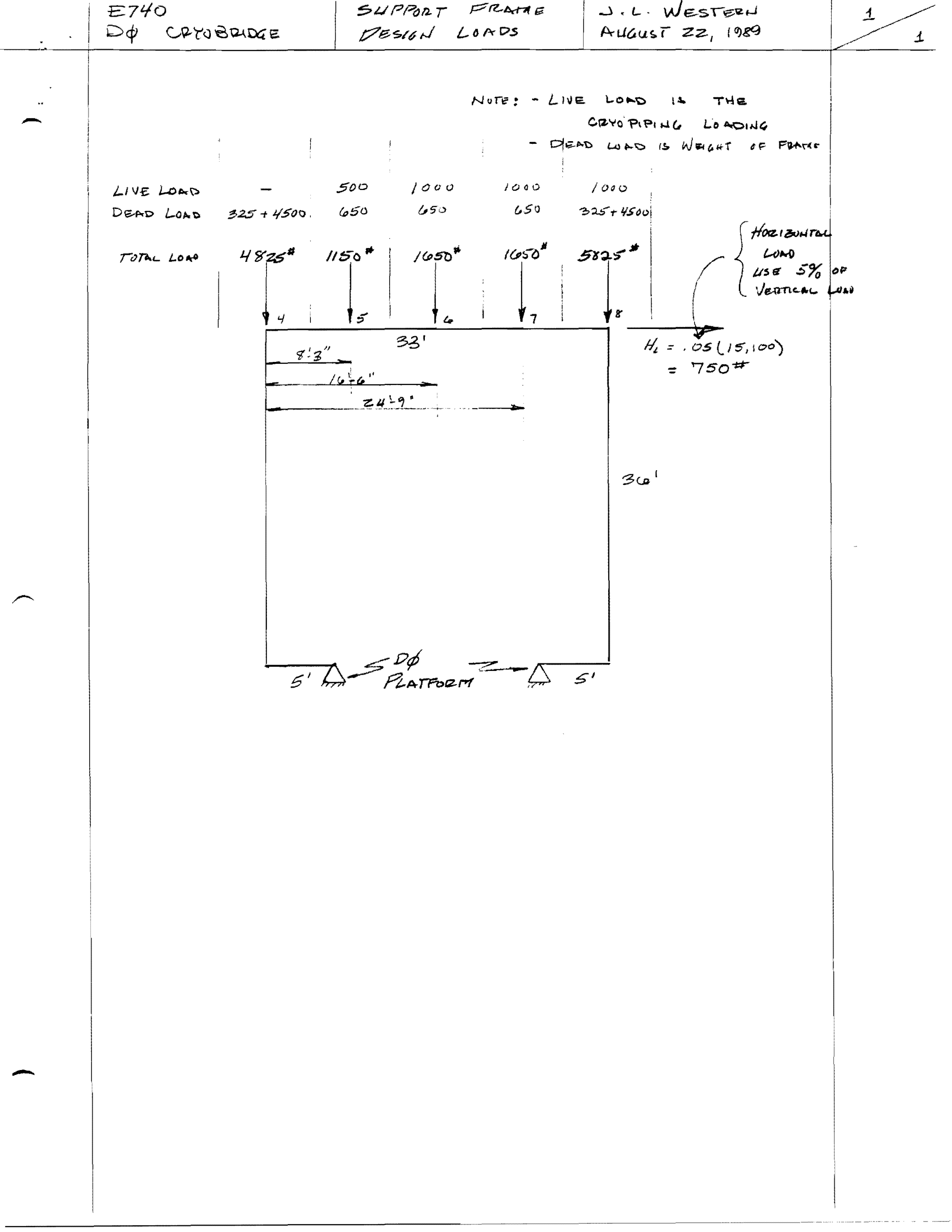


$E 740$

OQ CRYOBRIOLE

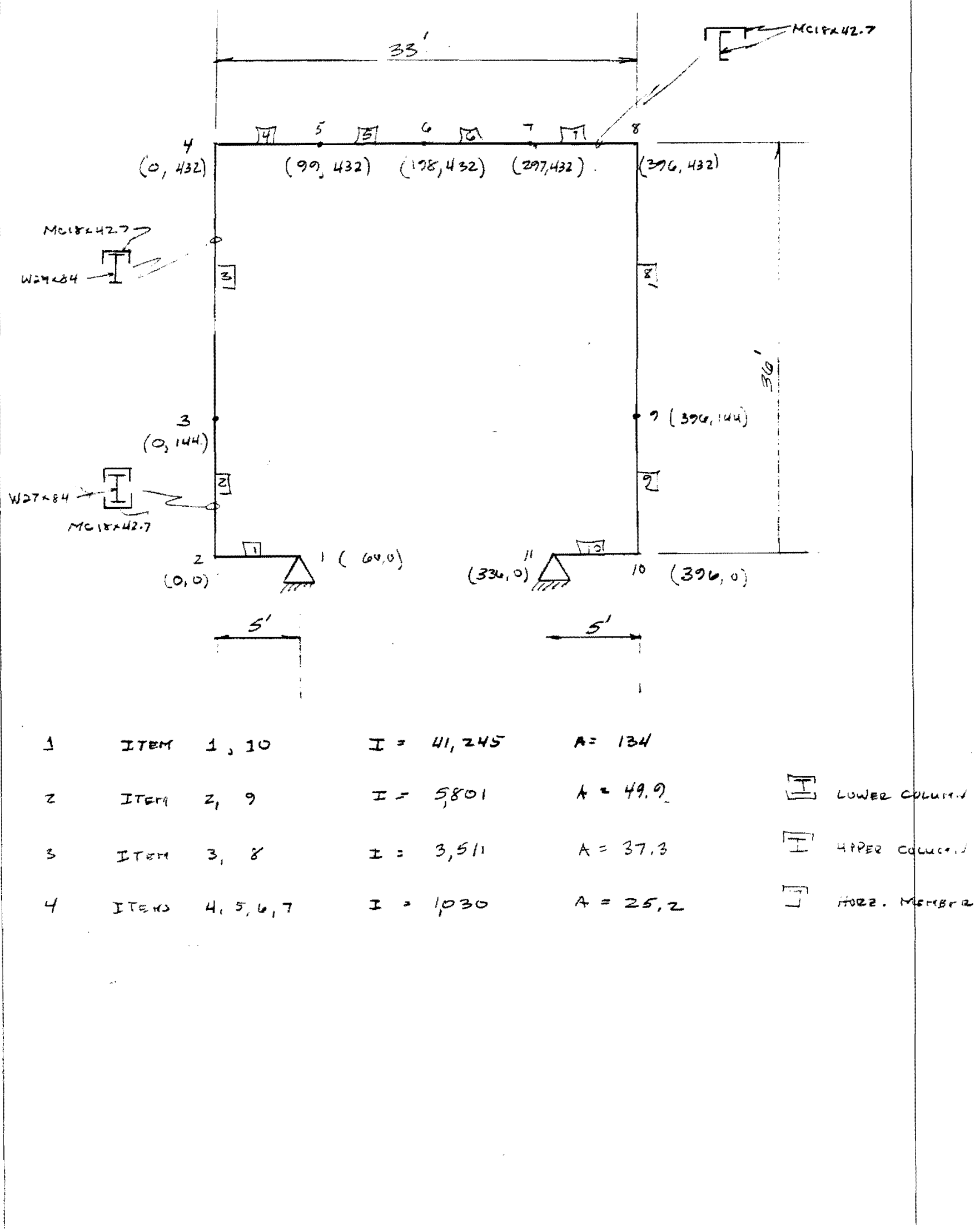




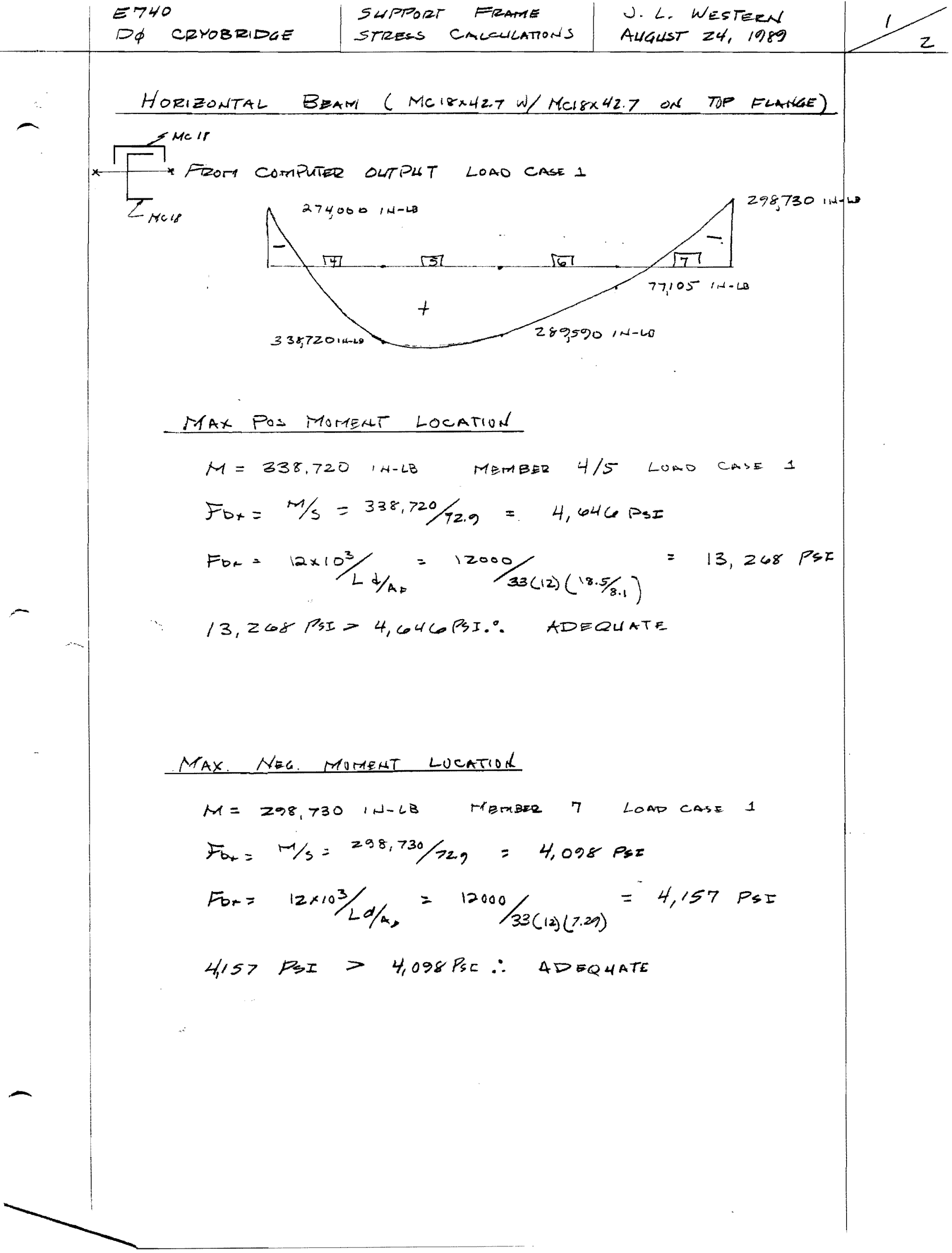




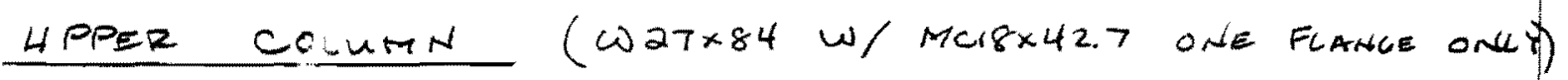

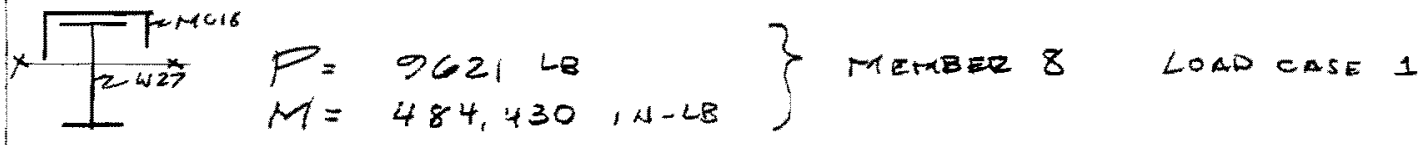

$F_{a}=P / A=9621 / 373=258 \cdot P_{\text {st }}$

$F_{u}=7,200$ Pse $\quad K L / r=(2.1) 24(12) / 4.2=144$

$F_{b x}=484,430 / 220=2,202 P_{55}$

$F_{D x}=12000 / 34(12)(4.19)=6,230 \mathrm{PSI}$

$$
\begin{aligned}
& F_{0+} / F_{0 x}+F_{0} / F_{a} \leq 1.0 \\
& 2,202 / 6,630+258 / 7200=.37 \leq 1.0 \\
& \therefore \text { ADEQUATE }
\end{aligned}
$$

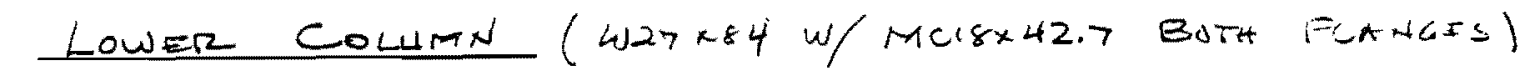
Trumis

$\left.\begin{array}{ll}2 W 27 & P=9421 \angle B \\ 1 & M=573280 \mathrm{IN}\end{array}\right\}$ MEBBER 9 LOAD CASE 1

$\frac{11}{\sum_{M C 16}}$

$F=P / A=9,621 / 40.9=193 P 512036$

$F_{a}=4360 \mathrm{ps} \quad \angle \mathrm{kL} / \mathrm{r}=2.036(12) / 4.9=185$

$F_{D x}=M / S=577,280 / 464=1,244 \mathrm{PSI}$

For $=12 \times 10^{\circ} / \angle \alpha / A_{2}=12000 / 30(12)(19 / 8,1)=11842$ PSI

$$
1,244 / 11,842+193 / 4,3600=1.0
$$

$\therefore A D E Q L A T E$ 


\begin{tabular}{|c|c|c|c|c|c|c|}
\hline$\angle P P E R$ & Colutin & $24^{\prime}$ & \multicolumn{2}{|c|}{ HEIGHT } & & \multirow[b]{2}{*}{ ONLK } \\
\hline W2- & $w /$ & $M C 18 x$ & 42.7 & ONE & FLAHGE & \\
\hline
\end{tabular}

Lower coluidi 36 ' Helatt

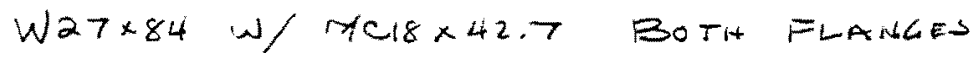

$$
\begin{aligned}
& K L / r_{y}=\frac{2.1(36)(12)}{4.9}=185 \leqslant 200 \\
& \text { NOTE: W27 } 68+\text { W/MCIS } 42.7 \text { ONE FAMGE ONCT } \\
& r L / r_{j}=\frac{2.1(36)(12)}{4.2}=216 \neq 200 \therefore \text { CHANASL ROTH }
\end{aligned}
$$


LPPER COLLMN

y

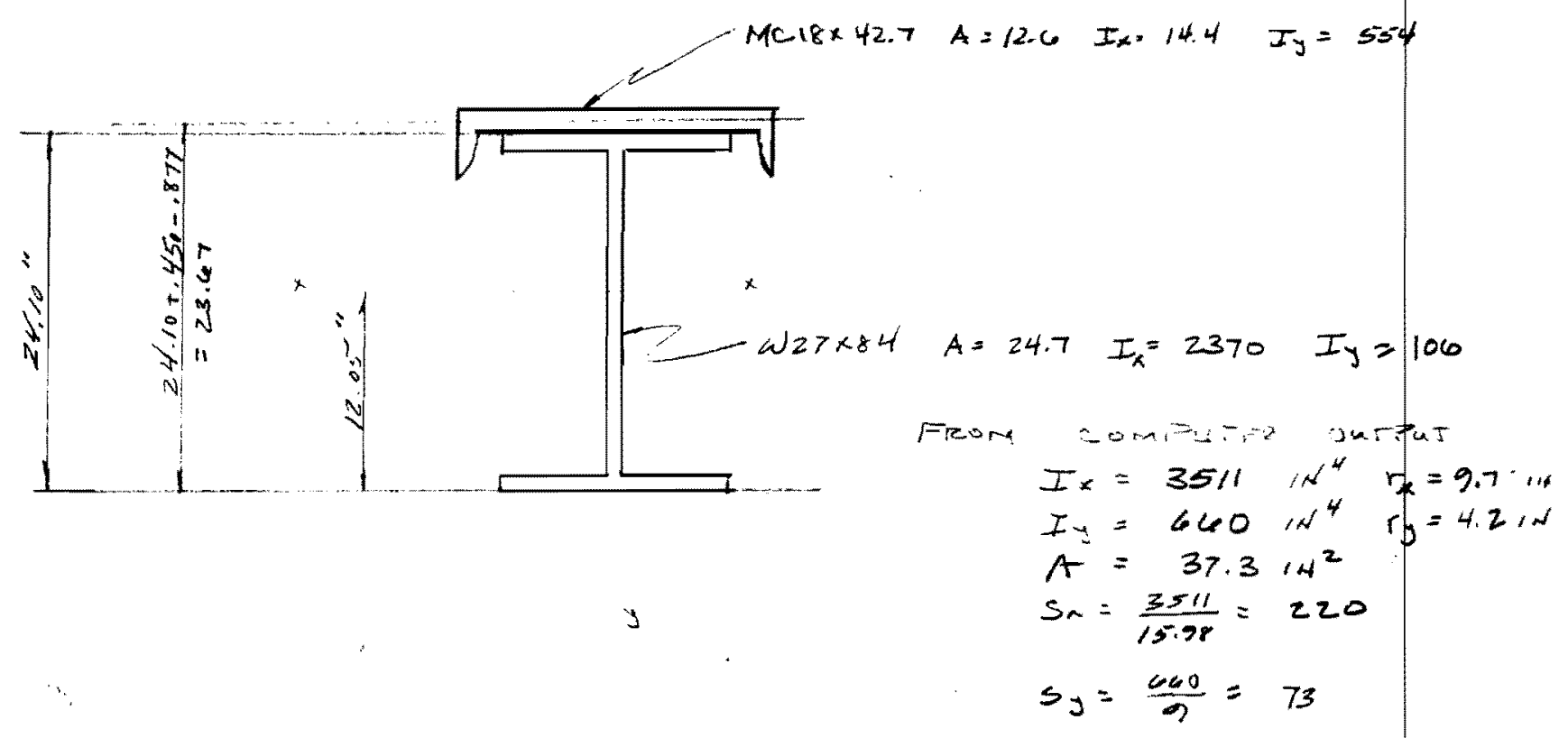

Lower Colums

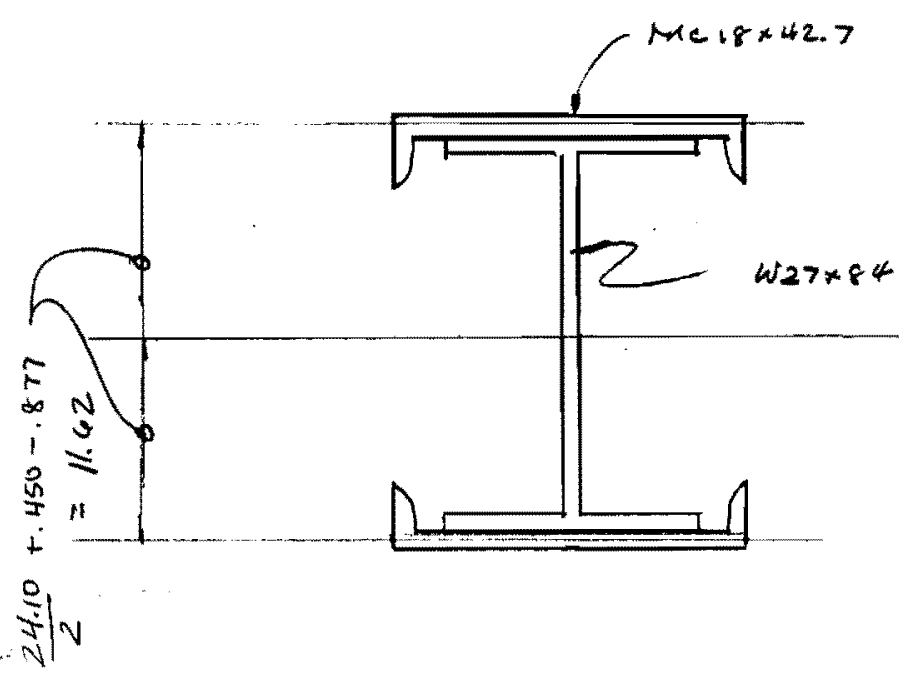

From computez ourpar

$I_{x}=5801 \mathrm{in}^{4} \quad r_{\alpha}=10.8$

$I_{y}=121414^{4} \quad r_{y} \cdot 4.9$

$A=49.9 \mathrm{in}^{2}$

$S_{x}=\frac{5801}{12.05+.45}=464$

$s_{j}=121 \% / 9,135$ 
Module A-1: Properties of Plane Sections

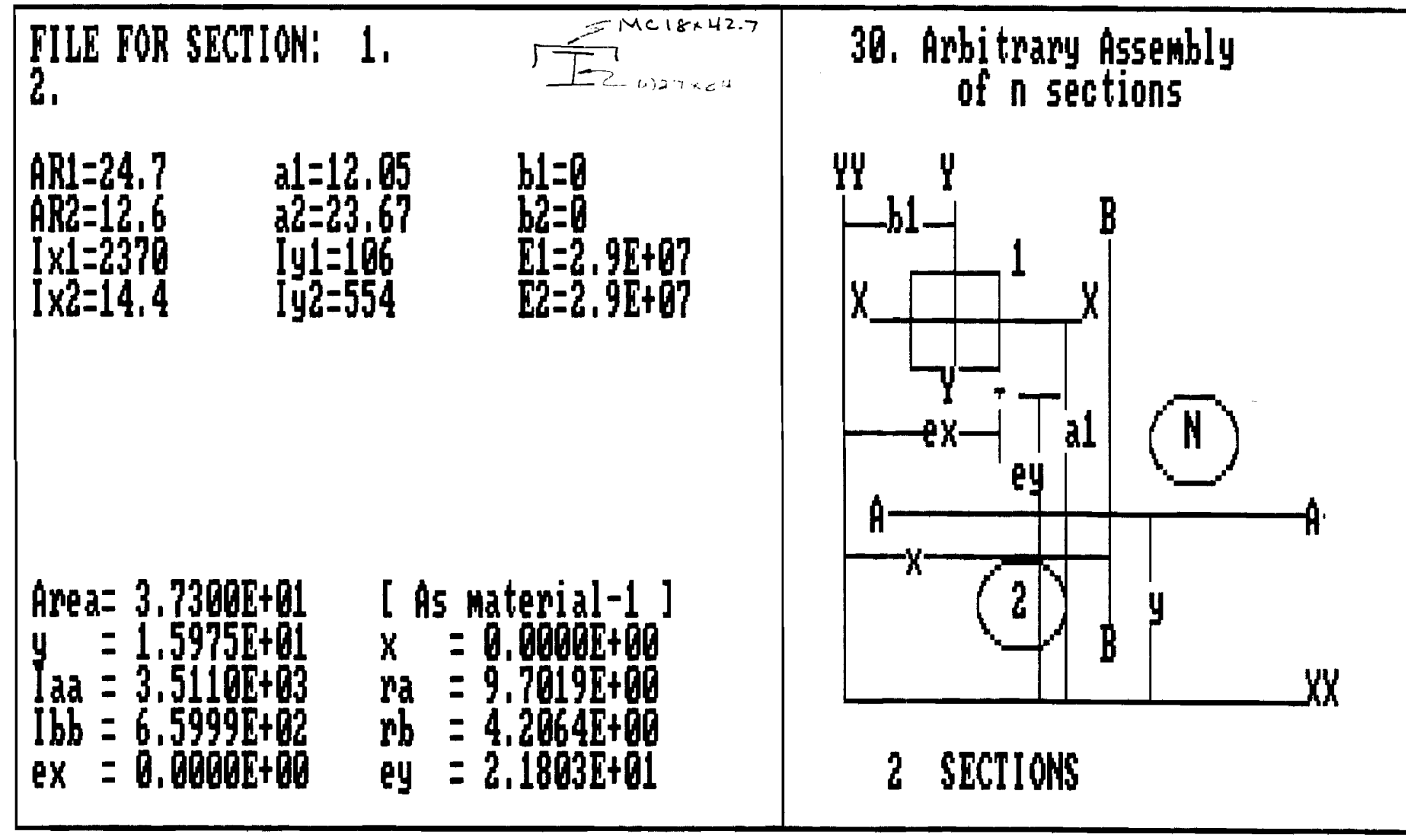

ENTER I7 FOR SECTION MENU

F2PRINI F3DISP

FTMENU TBREDO T9SAUE 
Module A-1: Properties of Plane Sections

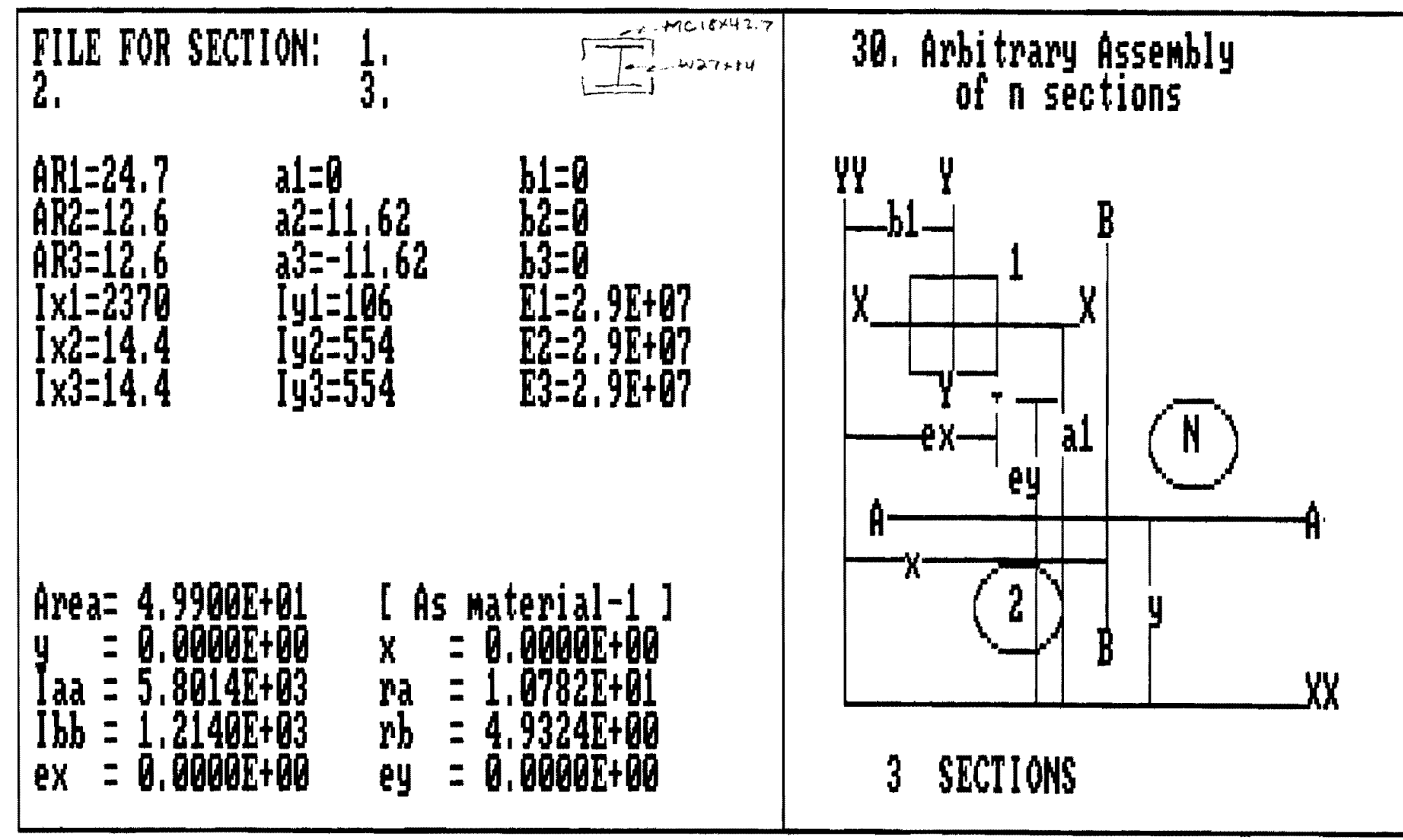

ENIER F7 FOR SECTION MENU

F2PRINT F3DISP

FTWENU FBREDO F9SAUE 
Module C-5: General Two-Dimensional Frames

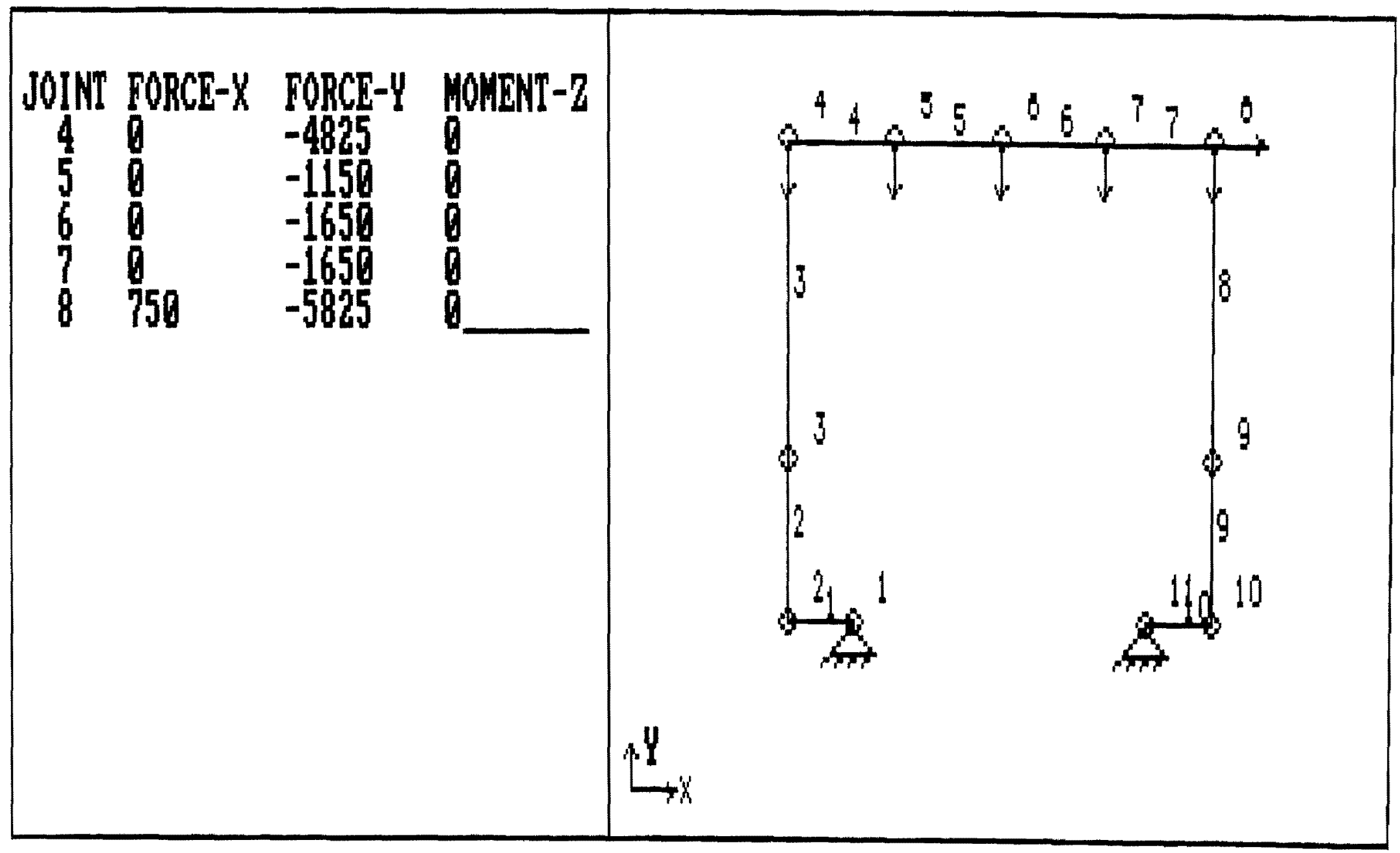

ENTER JOINT LOAD FOR 5 JOINTS OR

I7 FOR RESTRAINT SELECTION MENU

FTWEN FBREDO

FIOTOP

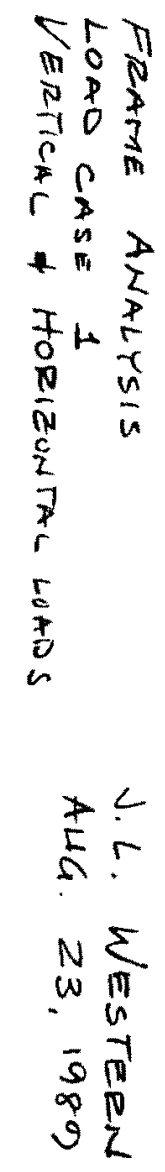


Module C-5: General Iwo-Dimensional Frames

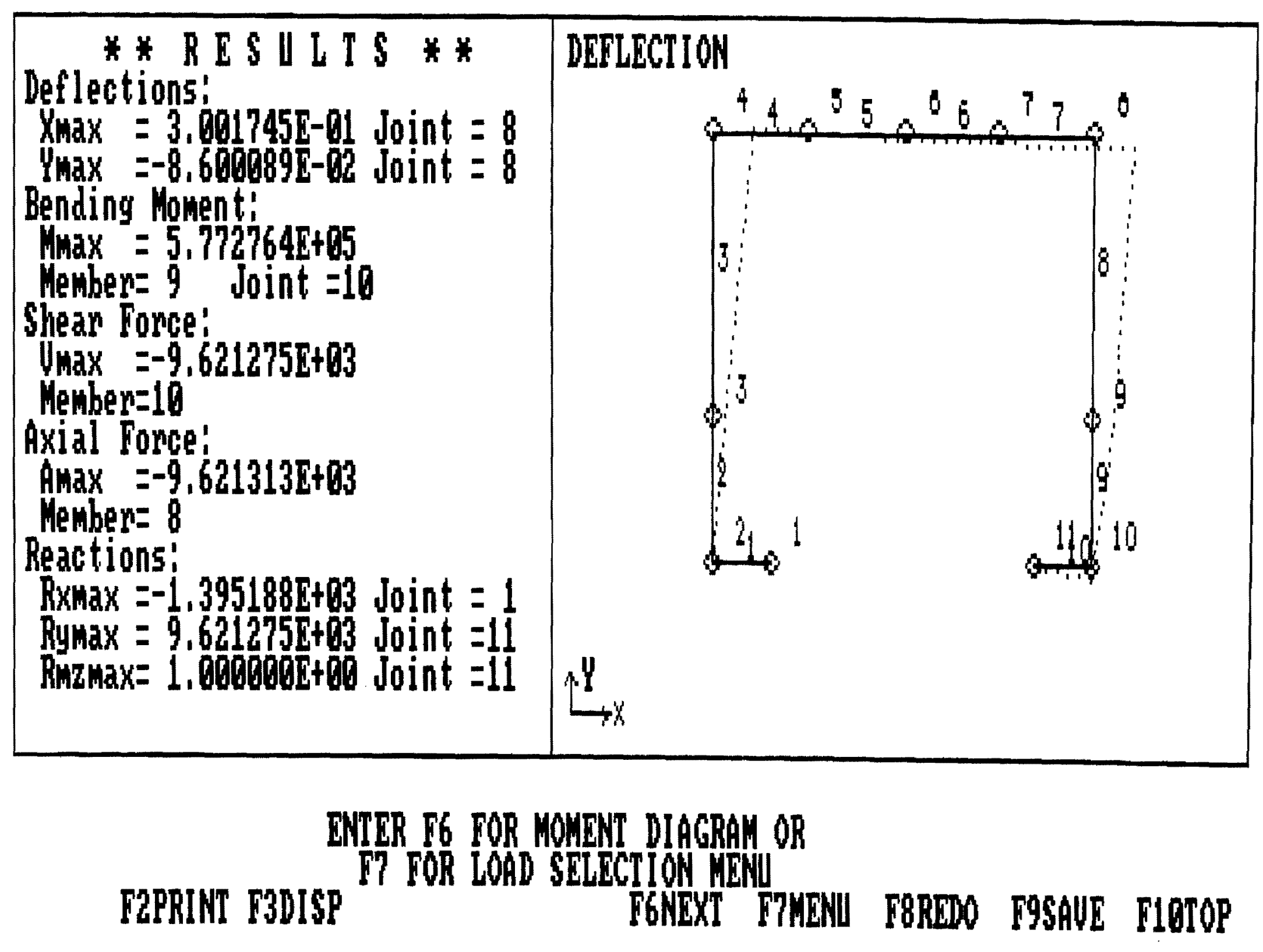


DE/CAASE Version 5.1

Module C-5 V3.1: General Two-Dimensional Frames

Developed by DVSE Inc. in cooperation with MSC

Copyright (C) 1985,1987 All Fights Feserved

$\ldots \ldots \ldots \ldots$ INFUT VAFI $\ldots \ldots$. $\ldots \ldots$. . . . . . .

NO. OF JOINTS

NO. OF JOINTS WITH FESTFATNTS $=1.1$

NO. OF DIFFEFENT MEMBEF FROFEFTIIES $=4$
NO. OF MEMEEFS

NO. OF JOINTS WITH APFLIED LOAD $=5$

$\begin{array}{cl}\text { JOINT } & X \text {-COOFD } \\ 1 & 6.000 E+01 \\ 2 & 0.000 E+00 \\ 3 & 0.000 E+00 \\ 4 & 0.000 E+00 \\ 5 & 9.900 E+01 \\ 6 & 1.980 E+02 \\ 7 & 2.970 E+02 \\ 0 & 3.960 E+02 \\ 9 & 3.960 E+02 \\ 10 & 3.960 E+02 \\ 11 & 3.360 E+02\end{array}$

MEMEER JOINT-1

$\begin{array}{rr}1 & 1 \\ 2 & 2 \\ 3 & 3 \\ 4 & 4 \\ 5 & 5 \\ 6 & 6 \\ 7 & 7 \\ 8 & 8 \\ 9 & 9 \\ 10 & 10\end{array}$

PROFERTY NO. 1
2
3

$Y-$ COORD

$0.000 \mathrm{E}+00$

$0.000 E+00$

1. $440 E+02$

4. $320 E+02$

4. $320 E+02$

4. $320 E+02$

$4.320 E+02$

4. $320 \mathrm{E}+02$

1. $440 E+02$

$0.000 E+00$

$0.000 E+00$
AFEA

$4.990 E+01$

4. $990 E+01$

$4.990 E+01$

4. $990 \mathrm{OE}+01$

\begin{abstract}
FORICE- $X$
$0.000 E+00$

$0.000 E+00$

$0.000 E+00$

$0.000 \mathrm{E}+00$

$0.000 E+00$

$0.000 E+00$

$0.000 E+00$

$7.500 E+02$

$0.000 E+00$

$0.000 E+00$

$0.000 E+00$
\end{abstract}

$$
\begin{array}{r}
\text { FOFCE-Y } \\
0.000 E+00 \\
0.000 E+00 \\
0.000 E+00 \\
-4.925 E+03 \\
-1.150 E+03 \\
-1.650 E+03 \\
-1.650 E+03 \\
-5.325 E+03 \\
0.000 E+00 \\
0.000 E+00 \\
0.000 E+00
\end{array}
$$

MOMENT $-Z$

$0.000 \mathrm{E}+00$

$0.000 \mathrm{E}+00$

$0.000 E+00$

$0.000 E+0 O$

$0.000 \mathrm{E}+00$

$0.000 E+00$

$0.000 E+00$

$0.000 \mathrm{E}+00$

$0.000 E+00$

$0.000 E+00$

\begin{tabular}{|c|c|c|c|c|c|c|c|c|c|}
\hline MAXIMUM & DEFLECTION & $\begin{array}{l}x \max \\
Y \max \end{array}$ & $\begin{array}{l}= \\
=\end{array}$ & $\begin{array}{r}3.0017 E-01 \\
-6.6001 E-02\end{array}$ & $\begin{array}{l}\text { JOINT } \\
\text { JOINT }\end{array}$ & $\begin{array}{l}= \\
=\end{array}$ & $\begin{array}{l}8 \\
8\end{array}$ & & \\
\hline MAXIMUM & BENDING MOME & & $=$ & $5.7728 E+05$ & MEMBEF & $=$ & 9 & JOINT & $=10$ \\
\hline MAXIMUM & AXIAL FORCE & & $=$ & $-9.6213 E+03$ & MEMEEF' & $=$ & 8 & & \\
\hline MAXIMUM & SHEAF FOFCE & & $=$ & $-9.6213 E+0.3$ & MIEMEEF & $=$ & 10 & & \\
\hline MAXIMUM & REACTION FX & & $=$ & $-1.3952 E+0$. & JOINT & $=$ & 1 & & \\
\hline MAXIMUM & REACTION FY & & $=$ & $9.6213 E+03$ & JOINT & $=$ & 1. 1 & & \\
\hline MAXIMUMI & FEACTION Fimz & & $=$ & $1.0000 E+00$ & JOINT & $=$ & 11 & & \\
\hline
\end{tabular}

$0.000 E+00$

JOINT-2 FROF NO.

$\begin{array}{rl}2 & 1 \\ 3 & 2 \\ 4 & 3 \\ 5 & 4 \\ 6 & 4 \\ 7 & 4 \\ 8 & 4 \\ 9 & 3 \\ 10 & 2 \\ 11 & 1\end{array}$

MOMENT OF INEFTIA

$5.801 E+03$
$5.801 E+03$
$5.801 E+03$
$5.801 E+03$
ELASTIC MODULUS

$2.900 E+07$

2. $900 \mathrm{E}+07$

2. $900 E+07$

$2.900 E+07$

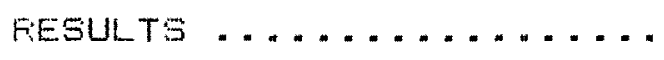

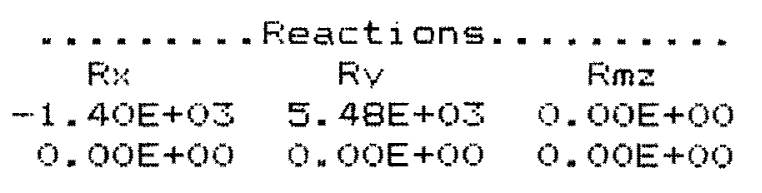




$\begin{array}{rr}3 & 9.2470 \mathrm{E}-02 \\ 4 & 2.9979 \mathrm{E}-0.1 \\ 5 & 2.9989 \mathrm{E}-01 \\ 6 & 2.9998 \mathrm{E}-01 \\ 7 & 3.0008 \mathrm{E}-01 \\ 9 & 3.0017 \mathrm{E}-01 \\ 9 & 1.559 \mathrm{E}-01 \\ 10 & -2.6734 \mathrm{E}-05 \\ 11 & 0.0000 \mathrm{E}+00\end{array}$

$$
\begin{array}{r}
2.8894 E-02 \\
2.7804 E-02 \\
-2.2986 E-02 \\
-5.5149 E-02 \\
-7.2028 E-02 \\
-8.6001 E-02 \\
-8.4086 E-02 \\
-8.3129 E-02 \\
0.0000 E+00
\end{array}
$$
$-7.2511 E-04$
$-5.9999 E-04$
$-4.1972 E-04$
$-2.5486 E-04$
$-1.2697 E-04$
$-1.9217 E-04$
$-8.6249 \mathrm{E}-04$
$-1.3169 \mathrm{E}-0 \mathrm{~S}$
$-1.4198 E-0 \mathrm{~S}$

$0.00 E+00$

$0.00 E+00$

$0.00 E+00$

$0.00 E+00$

$0.00 E+00$

$0.00 E+00$

$0.00 E+00$

$0.00 \mathrm{E}+00$

6. $45 E+02$

$0.00 E+00$

$0.00 E+00$

$0.00 E+00$

$0.00 E+00$

$0.00 E+00$

$0.00 E+00$

$0.00 E+00$

$0.00 E+00$

$0.00 E+00$

$0.00 E+00$

\begin{tabular}{|c|c|c|c|c|}
\hline EMBEF & AXIAL FORCE & SHEAF FOFICE & MOMENT JOINT-1 & MOMENT JOINT-Z \\
\hline 1 & $-1.3952 E+0.3$ & $5.4787 E+.03$ & $0.0000 E+00$ & $3.2872 E+05$ \\
\hline 2 & $-5.4788 E+0$. & $-1.3952 E+03$ & $-3.2872 E+05$ & $1.2782 \mathrm{E}+05$ \\
\hline 3 & $-5.4787 E+0.3$ & $-1.3952 E+02$ & $-1.2782 E+95$ & $-2.7400 E+05$ \\
\hline & $1.3755 E+0$. & $-6.5375 E+02$ & $2.7400 E+05$ & $-3,3872 E+05$ \\
\hline 5 & $1.3950 E+0$. & $4.9627 E+02$ & 3. 3B72E+05 & $-2.8959 E+055$ \\
\hline 6 & $1.3950 E+03$ & $2.14650+03$ & $2.8759 E+05$ & $7.7105 E+04$ \\
\hline 7 & $1.3950 E+03$ & $\therefore 7963 E+03$ & $7.7104 \mathrm{E}+04$ & $2.9873 E+05$ \\
\hline 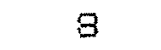 & $-9.6213 E+03$ & $6.4479 E+02$ & $-2.9873 E+05$ & $4.8443 E+05$ \\
\hline 9 & $-9.6213 E+0$. & $6.4479 E+02$ & $-4.8443 E+05$ & $5.7728 E+05$ \\
\hline 10 & $-6.4479 E+02$ & $-9.6213 E+03$ & $-5.7728 E+05$ & $-1.0000 E+00$ \\
\hline
\end{tabular}

$0.00 \mathrm{E}+00$

$0.00 E+00$

$0.00 E+00$

$0.00 E+00$

$0.00 E+00$

$0.00 E+00$

$9.62 E+03$

1. $00 E+00$ 
Module C-5: General Two-Dimensional Frames

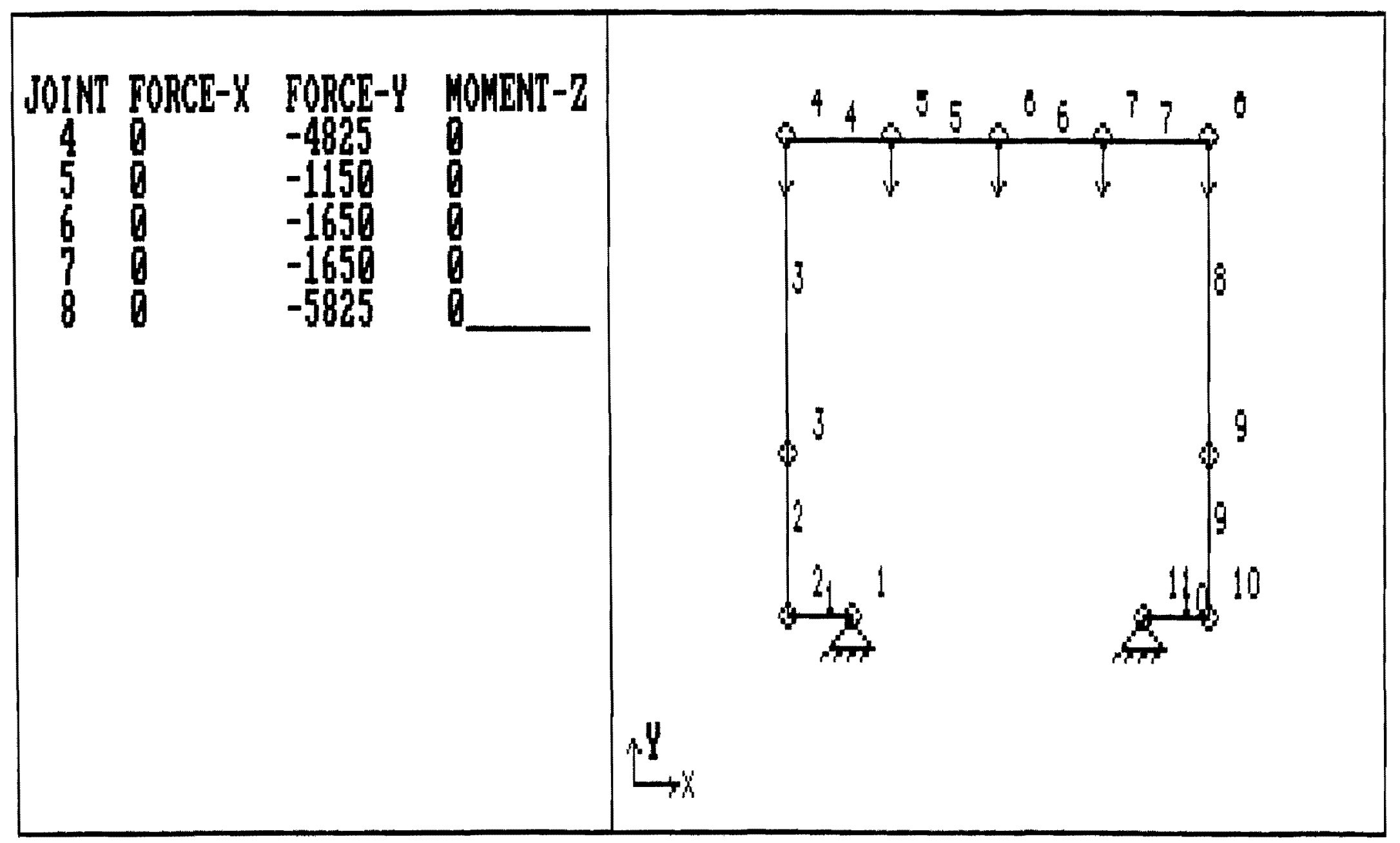

ENTER JOINT LOAD FOR 5 JOINTS OR

I7 TOR RESTRAINT SELECTION MENU

FTEND FBREDO

F10TOP

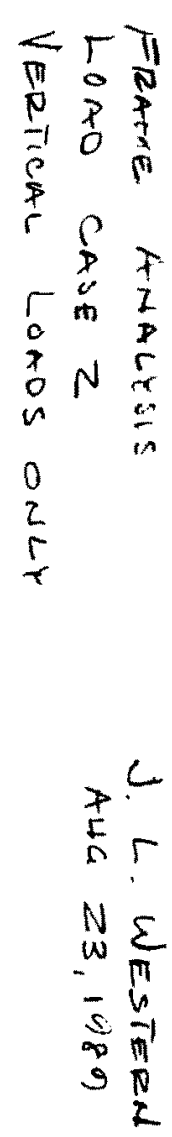


Module C-5: General Two-Dimensional Trames
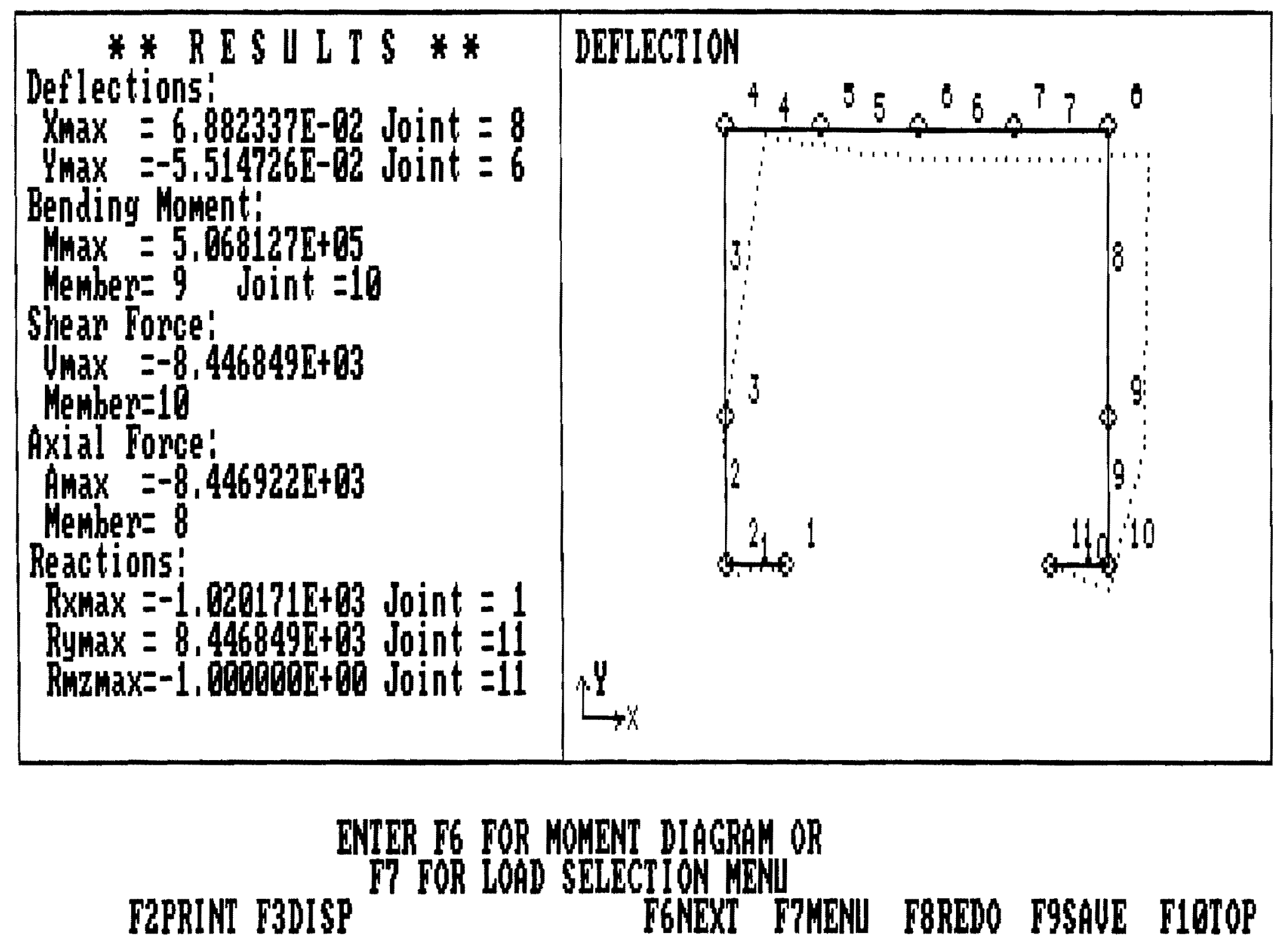
DE/CAASE Version 3.1

Module C-5 V. 1: General Two-Dimensional Frames DEVeloped by DVSE InC. in cooperation with MSC

Copyright (C) 1985,1987 All Fights Feserved

INFUT VARIAELES ............

NO. OF JGTNTS

$=11$ NO. OF MEMEERS

$=10$

NO. OF JOINTS WITH RESTFAINTS $=2$

NO. DF DIFFEFENT MEMEEF FROFERTIES $=4$

NO. OF JOINTS WITH APFLIED LOAD $=5$

$\begin{array}{cl}\text { JOINT } & X-\text { COORD } \\ 1 & 0.000 E+01 \\ 2 & 0.000 E+00 \\ 3 & 0.000 E+00 \\ 4 & 0.000 E+00 \\ 5 & 9.900 E+01 \\ 6 & 1.980 E+02 \\ 7 & 2.970 E+02 \\ 9 & 3.960 E+02 \\ 9 & 3.960 E+02 \\ 10 & 3.960 E+02 \\ 11 & 3.360 E+02\end{array}$

$Y-C D O R D$
$0.000 E+00$
$0.000 E+00$
$1.440 E+02$
$4.320 E+02$
$4.320 E+02$
$4.320 E+02$
$4.320 E+02$
$4.320 E+02$
$1.440 E+02$
$0.000 E+00$
$6.000 E+00$

BOUNDAFY

$\begin{array}{cc}\text { TYFE } & \text { FOFCE-X } \\ 3 & 0.000 E+00 \\ 0 & 0.000 E+00 \\ 0 & 0.000 E+00 \\ 0 & 0.000 E+00 \\ 0 & 0.000 E+00 \\ 0 & 0.000 E+00 \\ 0 & 0.000 E+00 \\ 0 & 0.000 E+00 \\ 0 & 0.000 E+00 \\ 0 & 0.000 E+00 \\ 3 & 0.000 E+00\end{array}$

FOFCE-Y

$0.000 E+00$

$0.000 E+00$

$0.000 E+00$

$-4.825 E+0 S$

$-1.150 E+05$

$-1.550 E+03$

$-1.650 E+05$

$-5.825 E+03$

$0.000 E+00$

$0.000 \mathrm{E}+0 \mathrm{O}$

$0.000 E+00$
MOMENT $-Z$

$0.000 E+00$

$0.000 E+00$

$0.000 E+00$

$0.000 E+00$

$0.000 \mathrm{E}+00$

$0.000 \mathrm{E}+00$

$0.000 E+00$

$0.000 E+00$

$0.000 \mathrm{E}+00$

$0.000 \mathrm{E}+00$

$0.000 E+00$

$\begin{array}{cccc}\text { MEMEEF } & 30 I N T-1 & \text { JOINT-2 } & \text { FFOF NO. } \\ 1 & 1 & 2 & 1 \\ 2 & 2 & 3 & 2 \\ 3 & 3 & 4 & 3 \\ 4 & 4 & 5 & 4 \\ 5 & 5 & 6 & 4 \\ 6 & 5 & 7 & 4 \\ 7 & 7 & 8 & 4 \\ 8 & 8 & 7 & 5 \\ 9 & 9 & 10 & 2 \\ 10 & 10 & 11 & 1\end{array}$

FROFERTY NO.

1
2
4

\begin{abstract}
AFEA
$4.970 E+01$

4. $990 E+01$

$4.990 E+01$

$4.990 \mathrm{E}+01$
\end{abstract}

MOMENT QF INERTIA

$$
\begin{aligned}
& 5.801 E+03 \\
& 5.801 E+03 \\
& 5.801 E+03 \\
& 5.801 E+03
\end{aligned}
$$

\begin{tabular}{|c|c|c|c|c|c|c|c|c|c|}
\hline MAXIMUM & DEFLECTION & $\begin{array}{l}X_{\max } \\
Y \max \end{array}$ & $\begin{array}{l}= \\
=\end{array}$ & $\begin{array}{r}6.8823 E-02 \\
-5.5147 E-02\end{array}$ & $\begin{array}{l}\text { JOINT } \\
\text { JOINT }\end{array}$ & $\begin{array}{l}= \\
=\end{array}$ & $\begin{array}{l}8 \\
6\end{array}$ & & \\
\hline MAXIMUM & BENDINE MOMEN & & $=$ & $5.0681 E+05$ & MEMEEF & $=$ & 9 & JUINT & $=10$ \\
\hline MAXIMUM & AXIAL FORCE & & $=$ & $-8.4 .469 E+03$ & MEMBEF: & $=$ & 8 & & \\
\hline MAXIMUM & SHEAF FOFCE & & $=$ & $-8.4468 E+03$ & MEMBEF & $=$ & 30 & & \\
\hline MAXIMUM & REACTION FX & & $=$ & $-1.0202 E+03$ & JOINT & $=$ & 1. & & \\
\hline MAXIMUM & FEEACTION RY & & $=$ & $8.4468 E+0.3$ & JOINT & $=$ & 11 & & \\
\hline MAXIMUM & REACTION RMz & & $=$ & $-1.0000 E+00$ & JOINT & $=$ & 11 & & \\
\hline
\end{tabular}

\begin{tabular}{|c|c|c|c|c|c|c|}
\hline & & Def & & & $E t$ & 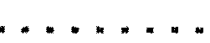 \\
\hline JOINT & $x$-DEFLECT ION & $Y$-DEFLECTION & Z-ROTATI IN & Fin & Fiv & $\operatorname{Finz}$ \\
\hline 1 & $.0000 E+00$ & $0.0000 E+00$ & $2.3651 E-04$ & $-1.02 E+03$ & $6.65 E+03$ & 1. $2 \square E-01$ \\
\hline 2 & $4.2299 E-05$ & $-1.2767 E-02$ & 1. $6533 E-04$ & $0.00 E+00$ & $0.00 E+00$ & $0.00 E+00$ \\
\hline
\end{tabular}

ELASTIC MODLLUS
$2.900 E+07$
$2.900 E+07$
$2.900 E+07$
$2.900 E+07$

FESULTS $\ldots \ldots \ldots \ldots$ 


$\begin{array}{rr}3 & -2.1827 \mathrm{E}-0 . \\ 4 & 6.8544 \mathrm{E}-02 \\ 5 & 6.8614 \mathrm{E}-02 \\ 6 & 6.3684 \mathrm{E}-02 \\ 7 & 6.8754 \mathrm{E}-02 \\ 8 & 0.8823 \mathrm{E}-02 \\ 7 & 0.1239 \mathrm{E}-02 \\ 10 & -4.2295 \mathrm{E}-05 \\ 11 & 0.0000 \mathrm{E}+00\end{array}$

$$
\begin{array}{r}
-1.3429 E-02 \\
-1.4753 E-02 \\
-4.0879 E-02 \\
-5.5147 E-02 \\
-5.4128 E-02 \\
-4.3427 E-02 \\
-4.1746 E-02 \\
-4.0905 E-02 \\
0.0000 E+00
\end{array}
$$

$$
\begin{array}{r}
-1.1347 E-04 \\
-2.9386 E-04 \\
-2.1618 E-04 \\
-6.5490 E-05 \\
7.6641 E-05 \\
1.1409 E-04 \\
-2.5058 E-04 \\
-6.2150 E-04 \\
-7.1188 E-04
\end{array}
$$
$0.00 E+00$

\begin{tabular}{|c|c|c|c|c|}
\hline MEMEEF & AXIAL FORCE & SHEAF FOFCE & MOMENT JOINT-1 & MOMENT JOINT-2 \\
\hline 1 & $-1.0202 E+03$ & $6.6531 E+03$ & $-1.2500 \mathrm{E}-01$ & $3.9919 E+05$ \\
\hline 2 & $-6.6531 E+03$ & $-1.0202 E+03$ & $-3.9919 E+05$ & $2.5228 E+05$ \\
\hline$\Xi$ & $-6.65 .1 E+0$. & $-1.0202 E+03$ & $-2.5228 E+05$ & $-4.1526 E+04$ \\
\hline 4 & $1.0201 E+03$ & $-1.3231 E+03$ & $4.1526 E+04$ & $-2.2251 E+05$ \\
\hline 5 & $1.0201 E+03$ & $-6.7813 E+02$ & $2.2251 E+05$ & $-2.8965 E+05$ \\
\hline 6 & $-1.0201 E+03$ & $9.7188 \mathrm{E}+02$ & $2.8965 E+05$ & $-1.9343 E+05$ \\
\hline 7 & $1.0201 E+03$ & $2.6219 E+0 E$ & $1.9543 E+05$ & $6.61 .8 E+04$ \\
\hline 8 & $-8.4469 E+03$ & 1.0201E+0S & $-6.6159 E+04$ & $3.5992 E+05$ \\
\hline 9 & $-8.4469 E+03$ & $1.0201 E+0 E$ & $-3.5992 E+05$ & $5.0681 E+05$ \\
\hline 10 & $-1.0201 E+03$ & $-8.4468 E+05$ & $.5 .0681 E+05$ & $1.0000+00$ \\
\hline
\end{tabular}
$0.00 E+00$
$0.0 O E+00$
$0.00 E+00$
$0.00 E+00$
$0.00 E+00$
$0.00 E+00$
$0.00 E+00$
$0.00 E+00$
$0.00 E+00$
$0.00 E+00$
$0.00 E+00$
$0.00 E+00$
$0.00 E+00$
$0.00 E+00$
$0.00 E+00$
$8.45 E+03-1.00 E+00$

$1.02 E+0 S$ 
Bending stress at Splice

$$
f_{b}=\frac{m_{c}}{I}=\frac{338720(13.655)}{759.8}=\frac{6087 \mathrm{psc}}{\frac{\text { OK }}{3}}
$$

Bolt Shear

* In a conservative approach we will assume the 12 bolts in the vertical 14" plate bear y the total load.

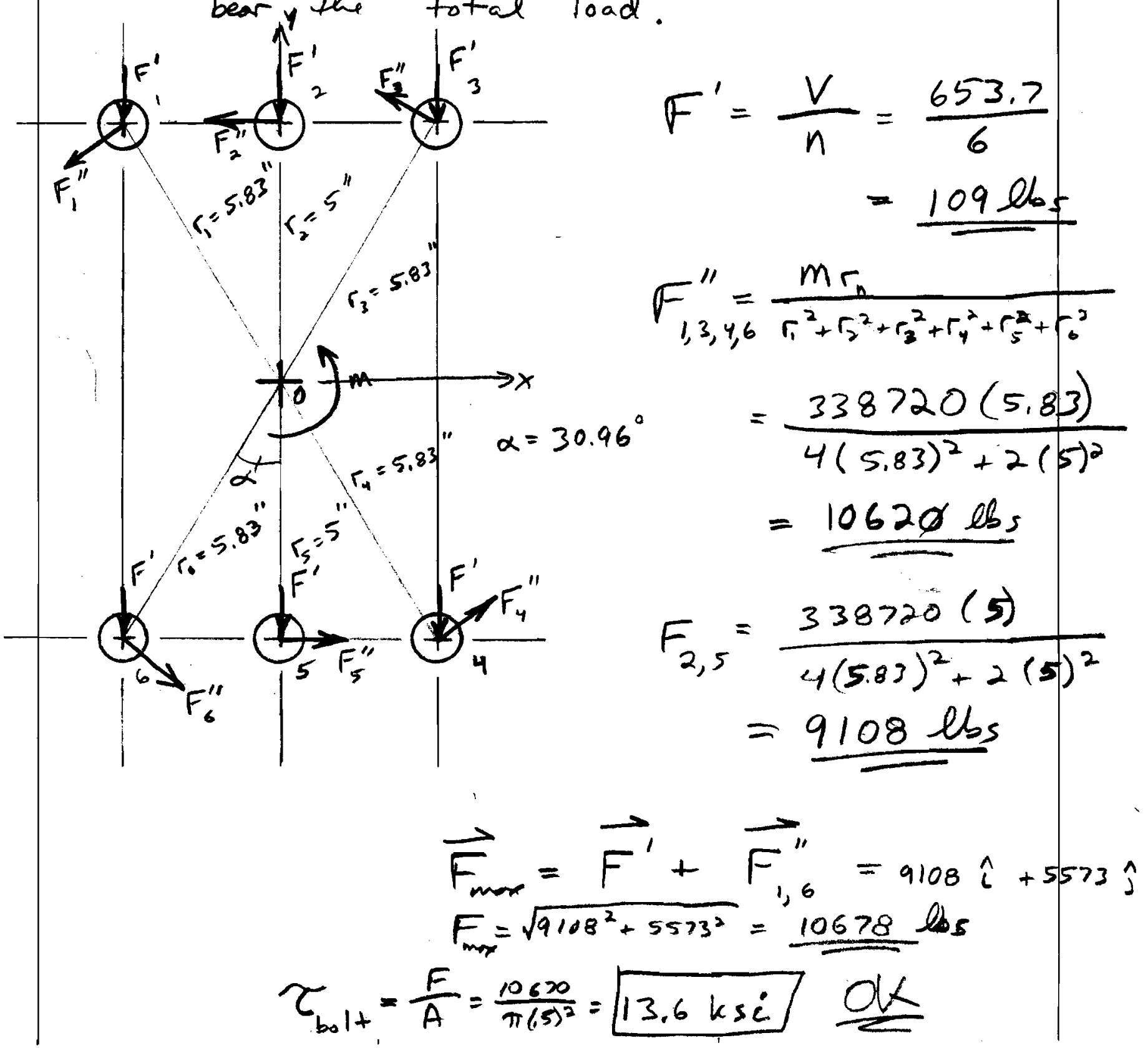




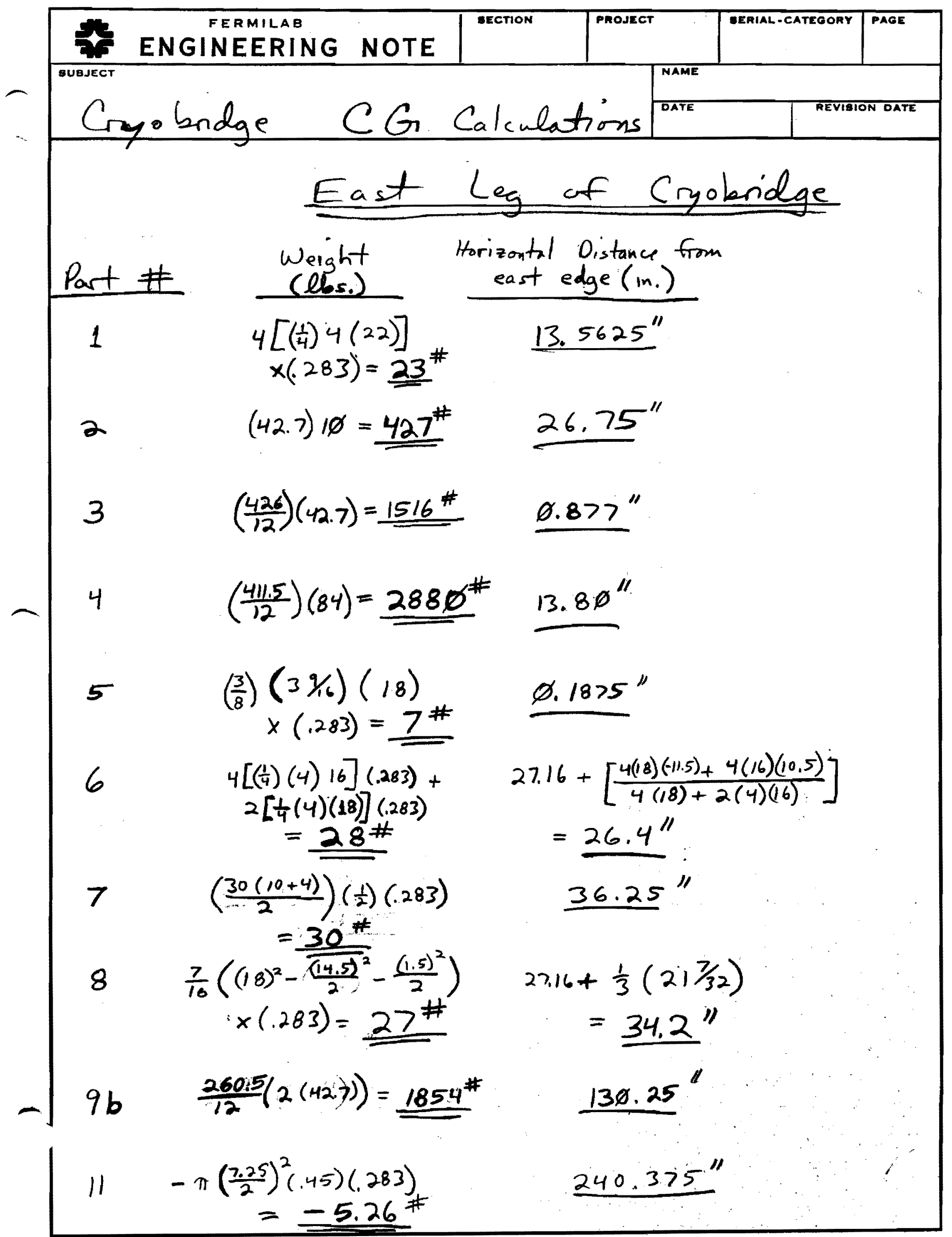




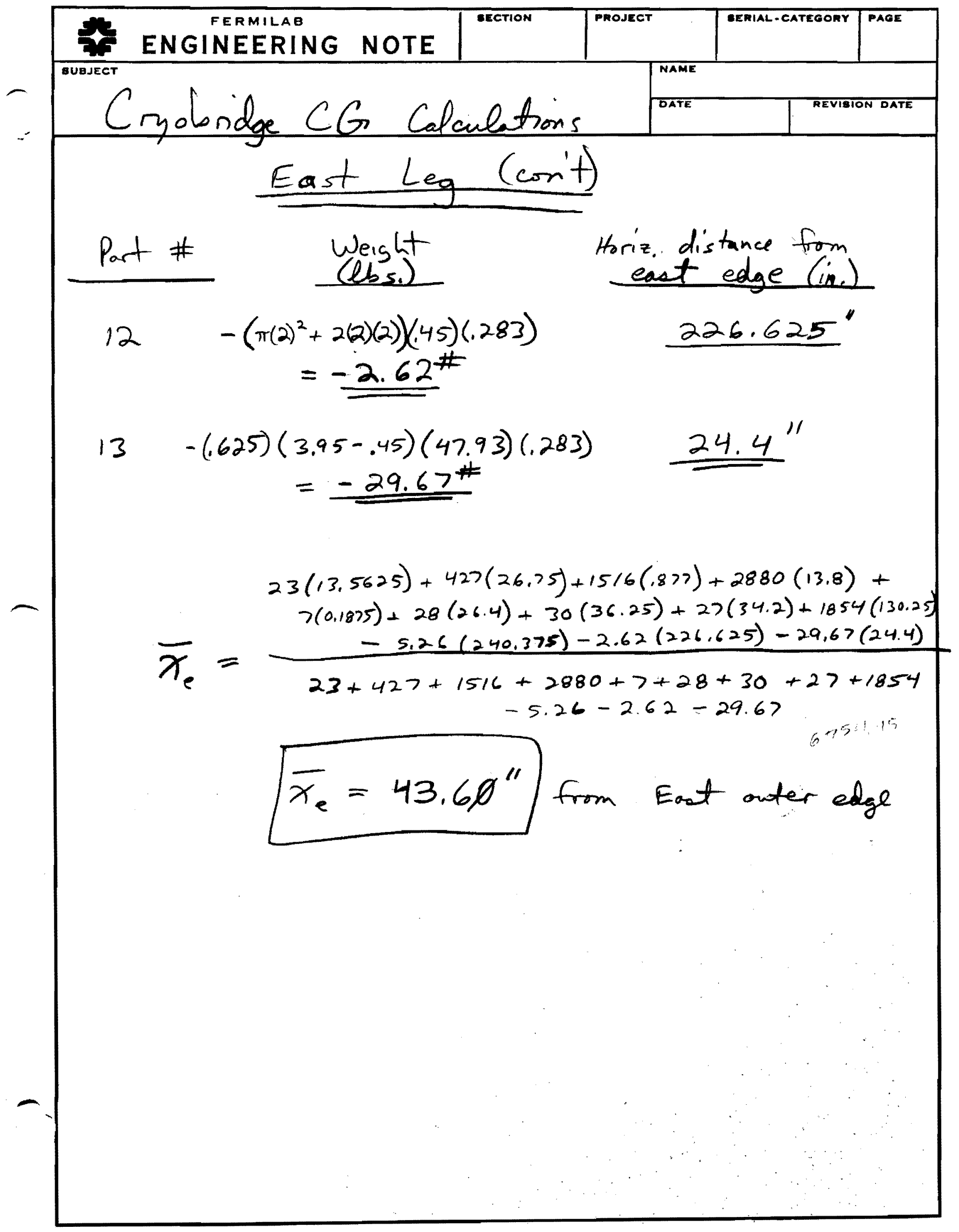




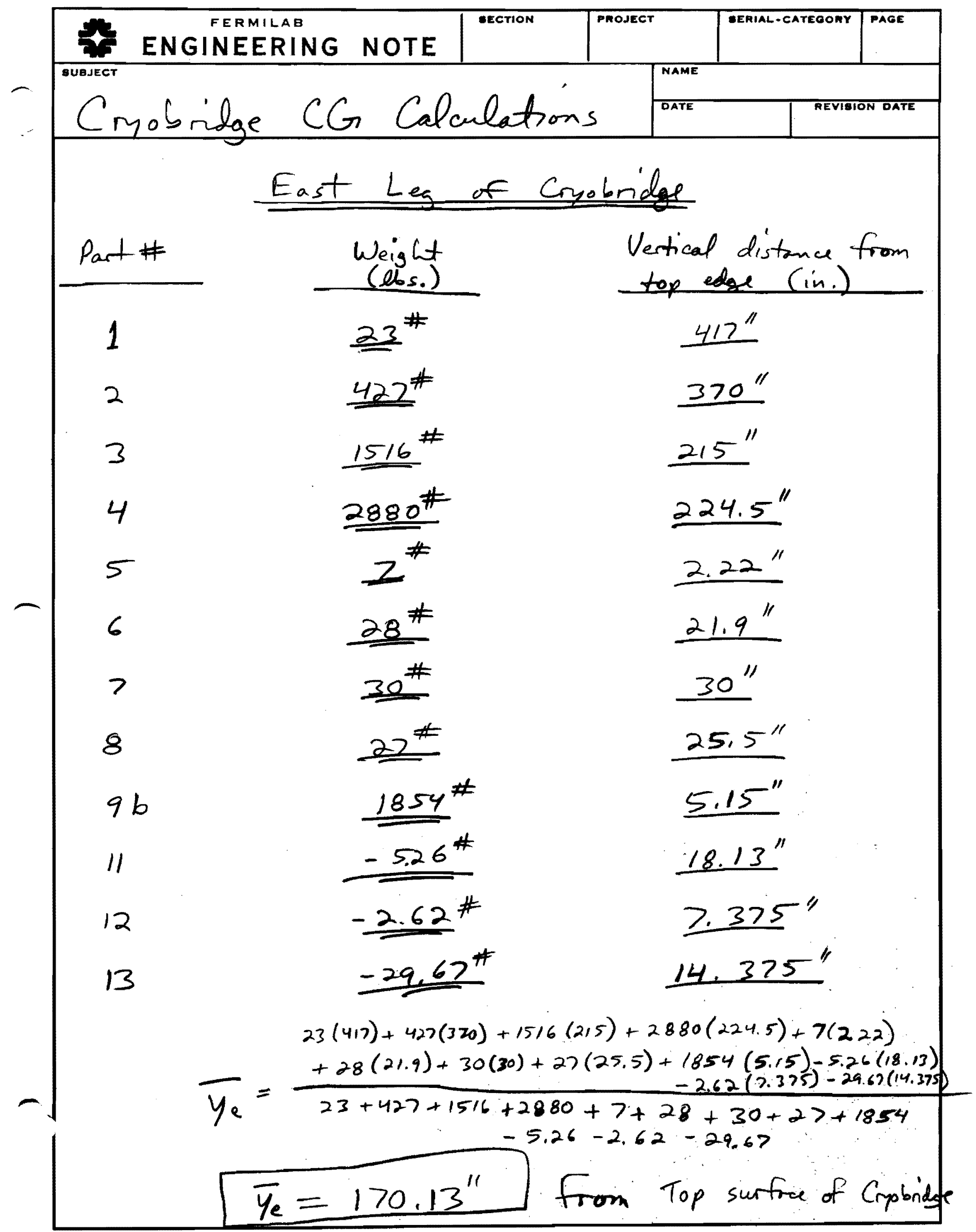




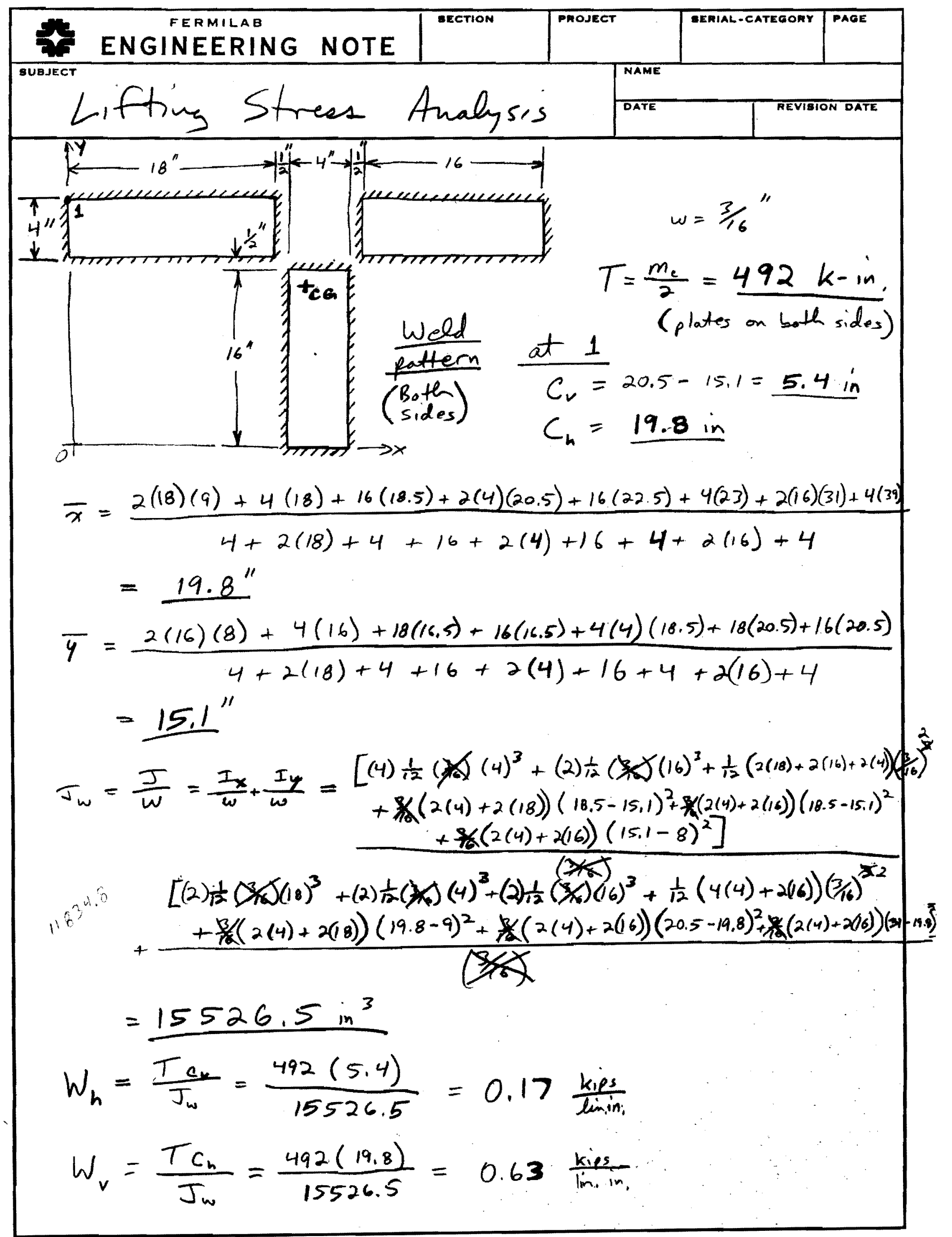




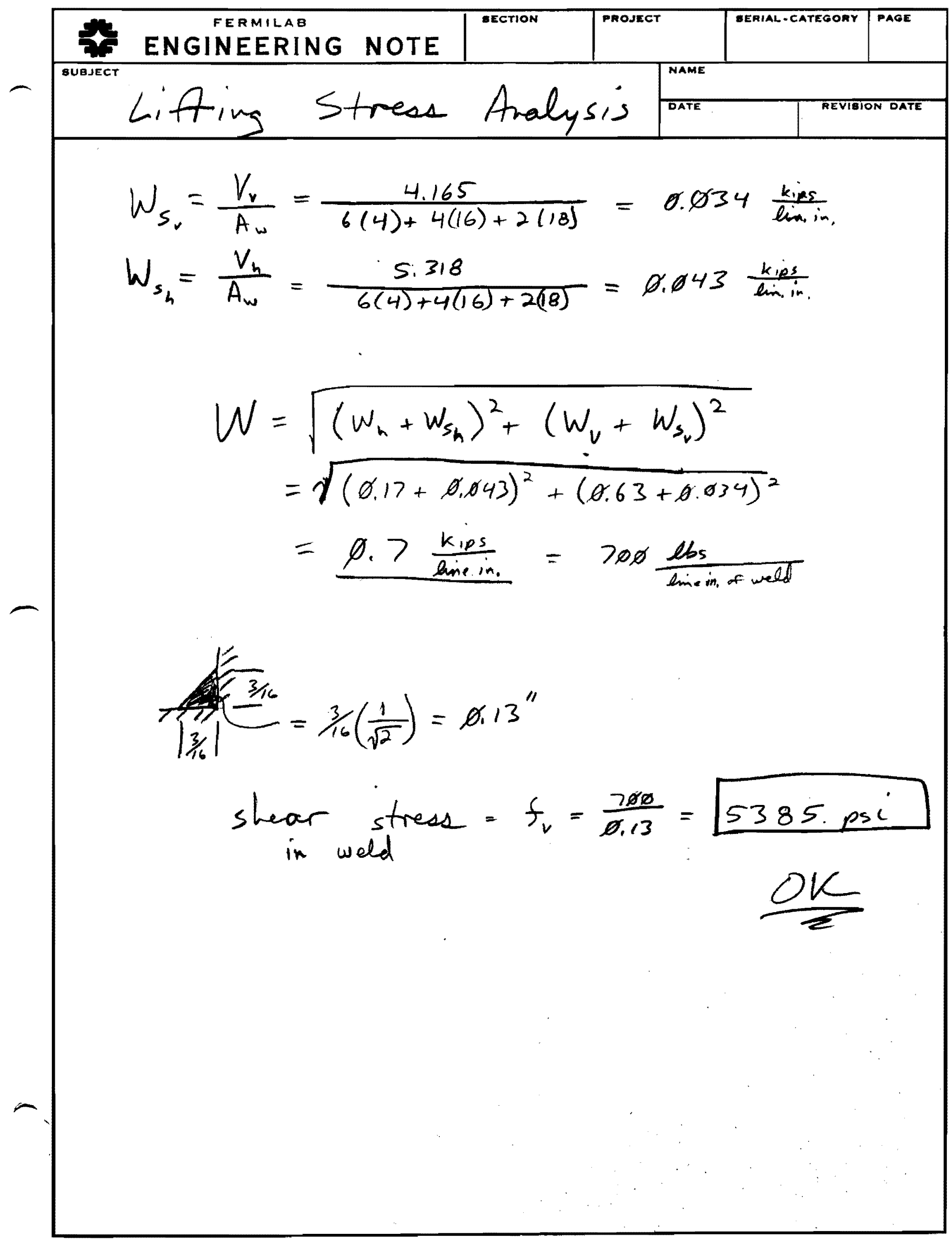



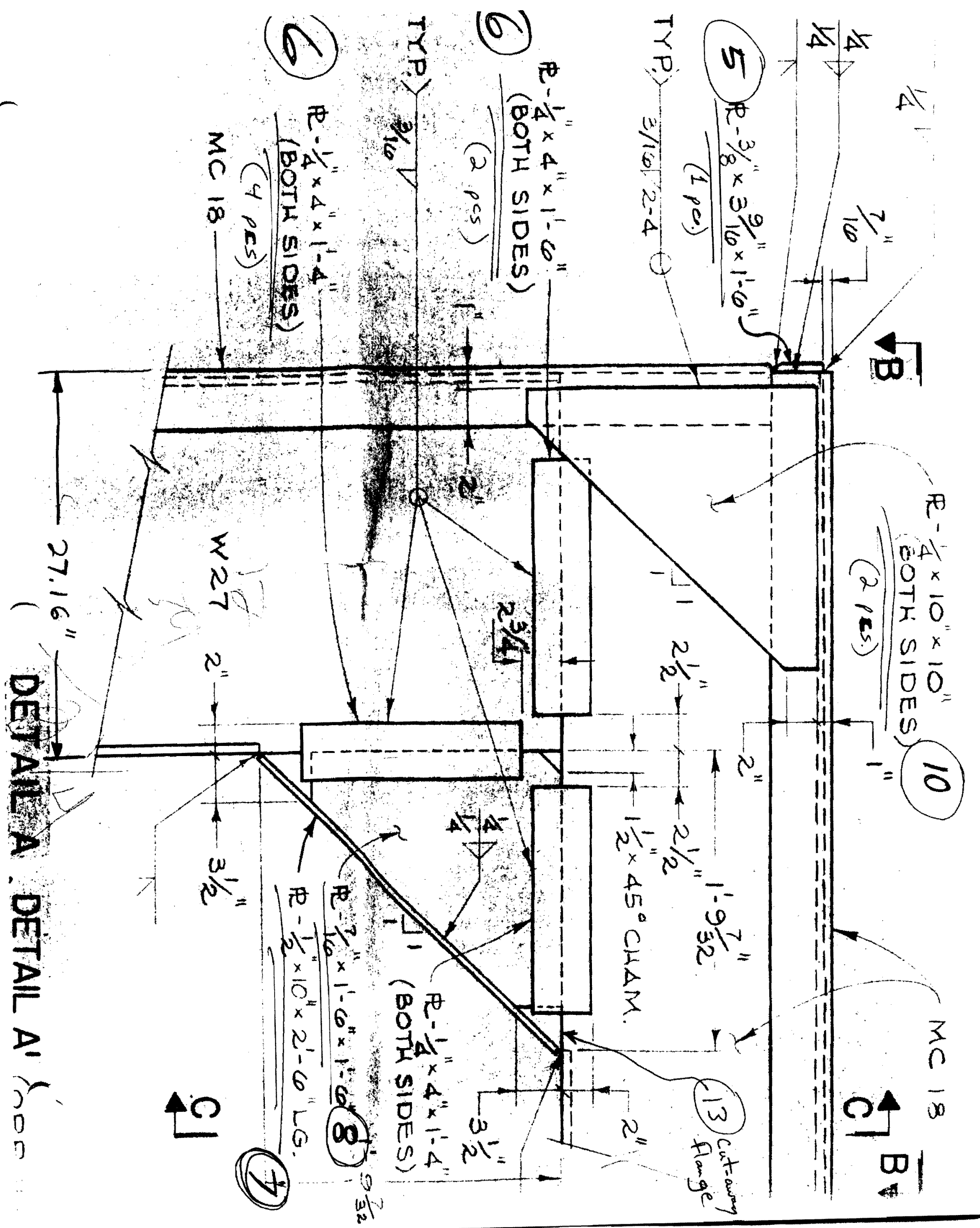
